\title{
Dirac quasinormal modes of new type black holes in new massive gravity
}

\author{
P. A. González ${ }^{1, a}$, Yerko Vásquez ${ }^{2, b}$ \\ ${ }^{1}$ Facultad de Ingeniería, Universidad Diego Portales, Avenida Ejército Libertador 441, Casilla 298-V, Santiago, Chile \\ 2 Departamento de Física, Facultad de Ciencias, Universidad de La Serena, Avenida Cisternas 1200, La Serena, Chile
}

Received: 16 May 2014 / Accepted: 4 July 2014 / Published online: 18 July 2014

(C) The Author(s) 2014. This article is published with open access at Springerlink.com

\begin{abstract}
We study a new type of black holes in threedimensional new massive gravity and we calculate analytically the quasinormal modes for fermionic perturbations for some special cases. Then we show that for these cases black holes of the new type are stable under fermionic field perturbations.
\end{abstract}

\section{Introduction}

In recent years, there has been a remarkable interest in the study of three-dimensional models of gravity. Apart from the BTZ black hole [1], which is a solution to the Einstein equations with a negative cosmological constant, much attention was given to topologically massive gravity (TMG), which is a generalization of three-dimensional GR that amounts to augment the Einstein-Hilbert action adding a Chern-Simons gravitational term [2-4]. Here, the propagating degree of freedom is a massive graviton. TMG also admits the BTZ (and other) black holes as exact solutions. The renewed interest on TMG relies on the possibility of constructing a chiral theory of gravity at a special point of the space of parameters [5].

On the other hand, Bergshoeff, Hohm, and Townsend (BHT) introduced another three-dimensional massive gravity theory, which is known as new massive gravity (NMG), being the action with the standard Einstein-Hilbert term with a specific combination of the scalar curvature square term and the Ricci tensor square one [6-10], and it is equivalent at the linearized level to the (unitary) Fierz-Pauli action for a massive spin-2 field [6]. The model in three dimensions is indeed unitary at tree level, but the corresponding model in higher dimensions is not, due to the appearance of non-unitary massless spin-2 modes [11]. Also, NMG admits

\footnotetext{
a e-mail: pablo.gonzalez@udp.cl

be-mail: yerko.vasquez@ufrontera.cl; yvasquez@userena.cl
}

warped AdS black holes [12], AdS waves [13,14], asymptotically Lifshitz black holes [15], gravitational solitons, kinks, and wormholes [16]; for further aspects of the BHT theory see [17-22]. Besides, asymptotically AdS and Lifshitz black holes in NMG dressed by a (non-)minimally coupled scalar field have been studied recently in [23]. It is worth to mention that TMG and NMG share common features, however, there are different aspects, one of them is the existence of the new type of black holes, for a specific combination of parameters in the NMG Lagrangian, which was discovered by BHT, and which are known as new type black holes.

The particular motivation of this work is to calculate the quasinormal modes (QNMs) for fermionic field perturbations in the background of the new type black holes in threedimensional new massive gravity and study the stability of these black holes under fermionic perturbations. The QNMs and their quasinormal frequencies (QNFs) are an important property of black holes and have a long history [24-29]. It is well known that the presence of event horizons dampens the vibration modes of a matter field that evolves perturbatively in the exterior region. In this way, the system is intrinsically dissipative, i.e., there is no temporary symmetry. In general, the oscillation frequencies are complex, therefore the system is not Hermitian. Nevertheless, the oscillation frequency of these modes is independent of the initial conditions and it only depends on the parameters (mass, charge, and angular momentum) and the fundamental constants (Newton constant and cosmological constant) that describe a black hole just like the parameters that define the test field. In threedimensional spacetime, the QNMs of the BTZ black hole have been studied in [30-32], and the QNMs for scalar field perturbations in the background of the new type black holes in NMG was studied in [33].

The QNMs give information as regards the stability of black holes under matter fields that evolve perturbatively in the exterior region of them, without backreacting on the metric. Also, the QNMs determine how fast a thermal state in 
the boundary theory will reach thermal equilibrium according to the AdS/CFT correspondence [34], where the relaxation time of a thermal state of the boundary thermal theory is proportional to the inverse of the imaginary part of the QNMs of the dual gravity background [35]. In the context of black hole thermodynamics, the QNMs allow the quantum area spectrum of the black hole horizon to be studied, as well as the mass and the entropy spectrum. In this regard, Bekenstein [36] was the first to propose the idea that in quantum gravity the area of black hole horizon is quantized, leading to a discrete spectrum which is evenly spaced. Then Hod [37] conjectured that the asymptotic QNF is related to the quantized black hole area, by identifying the vibrational frequency with the real part of the QNFs. However, it is not universal for every black hole background. Then Kunstatter [38] propose that the black hole spectrum can be obtained by imposing the Bohr-Sommerfeld quantization condition to an adiabatic invariant quantity involving the energy and the vibrational frequency. Furthermore, Maggiore [39] argued that in the large damping limit the identification of the vibrational frequency with the imaginary part of the QNF could lead to the Bekenstein universal bound. Then the consequences of these proposals were studied in several spacetimes; for instance, see [33], where the authors comment on the AdS/CFT correspondence and entropy/area spectrum for the new type of black holes. Besides, in [40-43] the authors discuss a connection between Hawking radiation and black hole quasinormal modes, which is important in the route to quantize gravity, because one can naturally interpret black hole quasinormal modes in terms of quantum levels.

The paper is organized as follows. In Sect. 2 we give a brief review of the new type of black holes in three-dimensional New Massive Gravity. In Sect. 3 we calculate the exact QNMs of fermionic perturbations for the new type black holes. Finally, our conclusions are presented in Sect. 4.

\section{New type black holes}

In this work we will consider matter distributions outside the event horizon of three-dimensional black holes, which are solutions of NMG and conformal gravity in three dimensions $[16,17,44]$. The action of NMG theory is given by

$S=\frac{1}{16 \pi G} \int \mathrm{d}^{3} x \sqrt{-g}\left(R-2 \lambda-\frac{1}{\mu^{2}} K\right)$,

where $K$ is given by the following quadratic terms of the curvature:

$K=R_{\mu \nu} R^{\mu \nu}-\frac{3}{8} R^{2}$,

where $R$ is the Ricci scalar, and $\lambda$ is the cosmological constant. In the special case $\mu^{2}=\lambda$ the theory admits the fol- lowing metric as a solution:

$\mathrm{d} s^{2}=-f(r) \mathrm{d} t^{2}+\frac{\mathrm{d} r^{2}}{f(r)}+r^{2} \mathrm{~d} \phi^{2}$,

where $f(r)=A r^{2}+B r+C$. In the case that $B \neq 0$ there is a curvature singularity due to the Ricci scalar diverging at $r=0$,

$R=-6 A-\frac{2 B}{r}$.

The metric (3) is conformally flat, and hence solves the field equations of conformal gravity in three dimensions too, as we mentioned. The parameter $A$ is proportional to the cosmological constant $\lambda, B$ is a kind of "gravitational hair" and $C$ is related to the mass of the spacetime. Note that depending on the values of the parameters $A, B$, and $C$ this metric may represent a black hole or not, the black holes solutions being known as new type black holes. The roots of $f(r)$ are

$r_{ \pm}=\frac{1}{2 A}\left(-B \pm \sqrt{B^{2}-4 A C}\right) ;$

for $r_{+}>0$ the metric represents new type black holes with an event horizon located at $r_{+}$. Also, in some cases there may exist a cosmological horizon $r_{-}$, with $r_{-}>r_{+}$. For $A>0$, the new type black holes are asymptotically anti-de Sitter, for $A<0$ they are asymptotically de Sitter, and for $A=0$ they are asymptotically locally flat. In the case $A=0$ the black holes are solutions of the theory that only contains the $K$ term in the Lagrangian. Additionally, for $A>0$ and $B=0$ this metric reduces to the non-rotating BTZ black hole.

\section{Dirac quasinormal modes of new type black holes}

The QNMs of fermionic perturbations in the background of the new type of black hole are given by the fermionic field solution to the Dirac equation in curved spacetime with suitable boundary conditions. This means that there are only ingoing waves on the event horizon and we will consider that the fermionic field vanishes at spatial infinity for asymptotically AdS black holes, known as Dirichlet boundary conditions, and that there are only outgoing waves at the cosmological horizon or at spatial infinity for asymptotically dS black holes and for asymptotically locally flat black holes, respectively. The Dirac equation is given by

$$
\left(\gamma^{\mu} \nabla_{\mu}+m\right) \psi=0 \text {, }
$$

where the covariant derivative is defined as

$\nabla_{\mu}=\partial_{\mu}+\frac{1}{2} \omega_{\mu}^{a b} J_{a b}$,

and the generators of the Lorentz group $J_{a b}$ are

$J_{a b}=\frac{1}{4}\left[\gamma_{a}, \gamma_{b}\right]$. 
The gamma matrices in curved spacetime $\gamma^{\mu}$ are defined by

$\gamma^{\mu}=e_{a}^{\mu} \gamma^{a}$

where $\gamma^{a}$ are the gamma matrices in flat spacetime. In order to solve the Dirac equation we use the diagonal vielbein

$e^{0}=\sqrt{f(r)} \mathrm{d} t, \quad e^{1}=\frac{\mathrm{d} r}{\sqrt{f(r)}}, \quad e^{2}=r \mathrm{~d} \phi$,

and, from the null torsion condition,

$\mathrm{d} e^{a}+\omega_{b}^{a} e^{b}=0$,

we obtain the spin connection

$\omega^{01}=\frac{f^{\prime}(r)}{2} \mathrm{~d} t, \quad \omega^{12}=-\sqrt{f(r)} \mathrm{d} \phi$.

Now, using the following representation of the gamma matrices:

$\gamma^{0}=i \sigma^{2}, \quad \gamma^{1}=\sigma^{1}, \quad \gamma^{2}=\sigma^{3}$,

where $\sigma^{i}$ are the Pauli matrices, and using the following ansatz for the fermionic field:

$\psi=\frac{e^{-i \omega t} e^{i \kappa \phi}}{\sqrt{f(r)^{1 / 2} r}}\left(\begin{array}{l}\psi_{1} \\ \psi_{2}\end{array}\right)$,

we arrive at the following coupled system of differential equations:

$\sqrt{f(r)} \psi_{1}^{\prime}+\frac{i \omega}{\sqrt{f(r)}} \psi_{1}-\frac{i \kappa}{r} \psi_{2}+m \psi_{2}=0$,

$\sqrt{f(r)} \psi_{2}^{\prime}-\frac{i \omega}{\sqrt{f(r)}} \psi_{2}+\frac{i \kappa}{r} \psi_{1}+m \psi_{1}=0$

where $m$ is the mass of the fermionic field $\psi$, which is minimally coupled to curvature. Decoupling the above system of equations we obtain the following equation for $\psi_{1}$ :

$$
\begin{aligned}
& -2 r^{2}(m r-i \kappa) f(r)^{2} \psi_{1}^{\prime \prime}+i r f(r)(2 \kappa f(r) \\
& \left.\quad+r(\kappa+i m r) f^{\prime}(r)\right) \psi_{1}^{\prime} \\
& \quad+\left(2\left(m^{3} r^{3}-i m^{2} r^{2} \kappa+m r \kappa^{2}-i \kappa^{3}-\kappa \omega r\right) f(r)\right. \\
& \left.-r^{2}(m r-i \kappa) \omega\left(2 \omega-i f^{\prime}(r)\right)\right) \psi_{1}=0 .
\end{aligned}
$$

Now, in order to obtain analytical solutions we will consider some special cases.

\subsection{Null angular momentum}

In this section we will consider the case $\kappa=0$. So, making the change of variables $y=1-\frac{r_{+}}{r}$ and defining $Q=\frac{r_{+}}{r_{-}}$,
Eq. (16) becomes

$$
\begin{aligned}
& \psi_{1}^{\prime \prime}(y)+\left(\frac{1 / 2}{y}+\frac{1}{y-1}+\frac{1 / 2}{y-1+Q}\right) \psi_{1}^{\prime}(z) \\
& +\left(-\frac{m^{2} Q / A}{y-1}+\frac{\frac{\omega^{2} Q^{2}}{(1-Q) A^{2} r_{+}^{2}}+\frac{i \omega Q}{2 A r_{+}}}{y}\right. \\
& \left.+\frac{-\frac{\omega^{2} Q^{3}}{(1-Q) A^{2} r_{+}^{2}}+\frac{i \omega Q^{2}}{2 A r_{+}}}{y-1+Q}\right) \frac{1}{y(y-1)(y-1+Q)} \psi_{1}(y)=0 .
\end{aligned}
$$

We note that this equation correspond to a Riemann's differential equation, whose general form is [45]

$$
\begin{aligned}
& \frac{\mathrm{d}^{2} w}{\mathrm{~d} z^{2}}+\left(\frac{1-\alpha-\alpha^{\prime}}{z-r}+\frac{1-\beta-\beta^{\prime}}{z-s}+\frac{1-\gamma-\gamma^{\prime}}{z-t}\right) \frac{\mathrm{d} w}{\mathrm{~d} z} \\
& +\left(\frac{\alpha \alpha^{\prime}(r-s)(r-t)}{z-r}+\frac{\beta \beta^{\prime}(s-t)(s-r)}{z-s}\right. \\
& \left.+\frac{\gamma \gamma^{\prime}(t-r)(t-s)}{z-t}\right) \frac{w}{(z-r)(z-s)(z-t)}=0,
\end{aligned}
$$

where $r, s, t$ are the singular points, and the exponents $\alpha, \alpha^{\prime}, \beta, \beta^{\prime}, \gamma, \gamma^{\prime}$ are subject to the condition

$\alpha+\alpha^{\prime}+\beta+\beta^{\prime}+\gamma+\gamma^{\prime}=1$.

The complete solution of (18) is denoted by the symbol

$w=P\left\{\begin{array}{cccc}r & s & t & \\ \alpha & \beta & \gamma & z \\ \alpha^{\prime} & \beta^{\prime} & \gamma^{\prime} & \end{array}\right\}$,

where the $P$ symbol denotes the Riemann $P$ function, which can be reduced to the hypergeometric function through

$$
\begin{aligned}
w= & \left(\frac{z-r}{z-s}\right)^{\alpha}\left(\frac{z-t}{z-s}\right)^{\gamma} \\
& \times P\left\{\begin{array}{cccc}
0 & \infty & 1 & \\
0 & \alpha+\beta+\gamma & 0 & \frac{(z-r)(t-s)}{(z-s)(t-r)} \\
\alpha^{\prime}-\alpha & \alpha+\beta^{\prime}+\gamma & \gamma^{\prime}-\gamma &
\end{array}\right.
\end{aligned}
$$

where the $P$ function is now the Gauss hypergeometric function.

\subsubsection{Asymptotically AdS new type black holes}

In this case $r_{+}>r_{-}(A>0)$. So, considering Eqs. (17) and (18) we can identify the regular singular points $r, s$, and $t$ as

$r=0, \quad s=1-Q, \quad t=1$ 
Therefore, the exponents are given by

$$
\begin{aligned}
& \alpha=-\frac{i \omega Q}{A r_{+}(Q-1)}, \quad \alpha^{\prime}=\frac{1}{2}+\frac{i \omega Q}{A r_{+}(Q-1)}, \\
& \beta=\frac{i \omega Q}{A r_{+}(Q-1)}, \quad \beta^{\prime}=\frac{1}{2}-\frac{i \omega Q}{A r_{+}(Q-1)}, \\
& \gamma=\frac{m}{\sqrt{A}}, \quad \gamma^{\prime}=-\frac{m}{\sqrt{A}},
\end{aligned}
$$

and the solution to Eq. (17) can be written as

$$
\begin{aligned}
& \psi_{1}(y)=C_{1}\left(\frac{y}{y-1+Q}\right)^{\alpha}\left(\frac{y-1}{y-1+Q}\right)^{\gamma} \\
& \quad{ }_{2} F_{1}\left(a, b, c, \frac{Q y}{y-(1-Q)}\right) \\
& +C_{2}\left(\frac{y}{y-1+Q}\right)^{\alpha^{\prime}}\left(\frac{y-1}{y-1+Q}\right)^{\gamma} \\
& \quad \times{ }_{2} F_{1}\left(a-c+1, b-c+1,2-c, \frac{Q y}{y-(1-Q)}\right),
\end{aligned}
$$

where we have defined the constants $a, b$, and $c$ as

$$
\begin{aligned}
& a=\alpha+\beta+\gamma, \\
& b=\alpha+\beta^{\prime}+\gamma, \\
& c=1+\alpha-\alpha^{\prime} .
\end{aligned}
$$

In the near horizon limit, the above expression behaves as

$$
\psi_{1}(y \rightarrow 0)=\widehat{C}_{1} y^{\alpha}+\widehat{C}_{2} y^{\alpha^{\prime}},
$$

with $\widehat{C}_{1}=C_{1}(-1)^{\gamma} /(-1+Q)^{\alpha+\gamma}$ and $\widehat{C}_{2}=C_{2}(-1)^{\gamma} /$ $(-1+Q)^{\alpha^{\prime}+\gamma}$. Now, we impose as boundary condition the requirement that classically nothing can escape from the event horizon. Note that $Q / A(Q-1)>0$, therefore we must take $C_{2}=0$ in order to have only ingoing waves at the horizon. So, the solution simplifies to

$$
\begin{aligned}
& \psi_{1}(y)=C_{1}\left(\frac{y}{y-1+Q}\right)^{\alpha}\left(\frac{y-1}{y-1+Q}\right)^{\gamma} \\
& \times_{2} F_{1}\left(a, b, c, \frac{Q y}{y-(1-Q)}\right) .
\end{aligned}
$$

Now, we implement boundary conditions at spatial infinity $y \rightarrow 1$. In order to do this, we employ the Kummer relations [45], which allow us to write the solution as

$$
\begin{aligned}
& \psi_{1}(y)=C_{1}\left(\frac{y}{y-1+Q}\right)^{\alpha}\left(\frac{y-1}{y-1+Q}\right)^{\gamma} \\
& \times \frac{\Gamma(c) \Gamma(c-a-b)}{\Gamma(c-a) \Gamma(c-b)} 2 F_{1} \\
& \times\left(a, b, a+b-c, 1-\frac{Q y}{y-(1-Q)}\right) \\
& +C_{1}(1-Q)^{\gamma^{\prime}-\gamma}\left(\frac{y}{y-1+Q}\right)^{\alpha}
\end{aligned}
$$

$$
\begin{aligned}
& \times\left(\frac{y-1}{y-1+Q}\right)^{\gamma^{\prime}} \frac{\Gamma(c) \Gamma(a+b-c)}{\Gamma(a) \Gamma(b)} \\
& \times{ }_{2} F_{1}\left(c-a, c-b, c-a-b+1,1-\frac{Q y}{y-(1-Q)}\right) .
\end{aligned}
$$

In the limit $y \rightarrow 1$, the above expression becomes

$$
\begin{aligned}
& \psi_{1}(y \rightarrow 1)=\widetilde{C}_{1}(1-y)^{\gamma} \frac{\Gamma(c) \Gamma(c-a-b)}{\Gamma(c-a) \Gamma(c-b)} \\
& +\widetilde{C}_{2}(1-Q)^{\gamma^{\prime}-\gamma}(1-y)^{\gamma^{\prime}} \frac{\Gamma(c) \Gamma(a+b-c)}{\Gamma(a) \Gamma(b)},
\end{aligned}
$$

with $\widetilde{C}_{1}=C_{1}(-1)^{\gamma} / Q^{\alpha+\gamma}$ and $\widetilde{C}_{2}=C_{1}(-1)^{\gamma^{\prime}} / Q^{\alpha+\gamma^{\prime}}$. So, imposing the requirement that the scalar field be null at spatial infinity, we can determine the quasinormal frequencies. For $m>0$ the second term of Eq. (31) blows up when $y \rightarrow 1$, unless we impose the condition $a=-n$ or $b=-n$, with $n=0,1,2, \ldots$, and these conditions give the following QNFs:

$\omega=-i \frac{A\left(r_{+}-r_{-}\right)}{4}\left(1+2 n+\frac{2 m}{\sqrt{A}}\right)$.

Due to the imaginary part of the QNFs being negative the asymptotically $A d S$ new type black holes are stable under fermionic perturbations, at least for the mode with the lowest angular momentum. Also, in a similar way the quasinormal frequencies associated to $\psi_{2}$ can be obtained; note that $\psi_{2}$ satisfies a similar equation to $\psi_{1}$ but making the changes $\kappa \rightarrow-\kappa$ and $\omega \rightarrow-\omega$.

\subsubsection{Asymptotically $d S$ new type black holes}

In this case, besides the event horizon $r_{+}$, we have a cosmological horizon $r_{-}$, where $r_{-}>r_{+}(A<0)$. So, considering Eqs. (17) and (18) we can identify the regular singular points $r, s$, and $t$ as

$r=0, \quad s=1, \quad t=1-Q$.

Therefore, the exponents are given by

$\alpha=-\frac{i \omega Q}{A r_{+}(Q-1)}, \quad \alpha^{\prime}=\frac{1}{2}+\frac{i \omega Q}{A r_{+}(Q-1)}$,

$\beta=\frac{m}{\sqrt{A}}, \quad \beta^{\prime}=-\frac{m}{\sqrt{A}}$,

$\gamma=\frac{i \omega Q}{A r_{+}(Q-1)}, \quad \gamma^{\prime}=\frac{1}{2}-\frac{i \omega Q}{A r_{+}(Q-1)}$,

and the solution to Eq. (17) can be written as

$$
\begin{gathered}
\psi_{1}(y)=C_{1}\left(\frac{y}{y-1}\right)^{\alpha}\left(\frac{y-1+Q}{y-1}\right)^{\gamma} \\
\times_{2} F_{1}\left(a, b, c,-\frac{Q y}{(y-1)(1-Q)}\right)
\end{gathered}
$$




$$
\begin{aligned}
& +C_{2}\left(\frac{y}{y-1}\right)^{\alpha^{\prime}}\left(\frac{y-1+Q}{y-1}\right)^{\gamma} \\
& \times{ }_{2} F_{1}\left(a-c+1, b-c+1,2-c,-\frac{Q y}{(y-1)(1-Q)}\right),
\end{aligned}
$$

where we have defined the constants $a, b$, and $c$ as

$a=\alpha+\beta+\gamma$,
$b=\alpha+\beta^{\prime}+\gamma$,
$c=1+\alpha-\alpha^{\prime}$.

In the near horizon limit, the above expression behaves as

$\psi_{1}(y \rightarrow 0)=\widehat{C}_{1} y^{\alpha}+\widehat{C}_{2} y^{\alpha^{\prime}}$,

with $\widehat{C}_{1}=C_{1}(-1+Q)^{\gamma} /(-1)^{\alpha+\gamma}$ and $\widehat{C}_{2}=C_{2}(-1+$

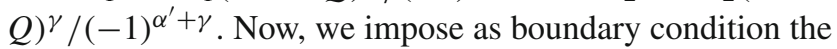
requirement that classically nothing can escape from the event horizon. Note that $Q / A(Q-1)>0$ as in the case 1 , therefore we must take $C_{2}=0$ in order to have only ingoing waves at the horizon. So, the solution simplifies to

$$
\begin{gathered}
\psi_{1}(y)=C_{1}\left(\frac{y}{y-1}\right)^{\alpha}\left(\frac{y-1+Q}{y-1}\right)^{\gamma} \\
\times{ }_{2} F_{1}\left(a, b, c,-\frac{Q y}{(y-1)(1-Q)}\right) .
\end{gathered}
$$

Now, we implement boundary conditions at the cosmological horizon $y \rightarrow 1-Q$. In order to do this, we employ the Kummer relations [45], which allow us to write the solution as

$$
\begin{aligned}
& \psi_{1}(y)=C_{1}\left(\frac{y}{y-1}\right)^{\alpha}\left(\frac{y-1+Q}{y-1}\right)^{\gamma} \frac{\Gamma(c) \Gamma(c-a-b)}{\Gamma(c-a) \Gamma(c-b)} \\
& \times{ }_{2} F_{1}\left(a, b, a+b-c, 1+\frac{Q y}{(y-1)(1-Q)}\right) \\
& +C_{1}\left(\frac{1}{1-Q}\right)^{\gamma^{\prime}-\gamma}\left(\frac{y}{y-1}\right)^{\alpha}\left(\frac{y-1+Q}{y-1}\right)^{\gamma^{\prime}} \\
& \times \frac{\Gamma(c) \Gamma(a+b-c)}{\Gamma(a) \Gamma(b)} \\
& \times{ }_{2} F_{1}\left(c-a, c-b, c-a-b+1,1+\frac{Q y}{(y-1)(1-Q)}\right) .
\end{aligned}
$$

In the limit $y \rightarrow 1-Q$, the above expression becomes

$$
\begin{aligned}
& \psi_{1}(y \rightarrow 1)=\widetilde{C}_{1}(1-Q-y)^{\gamma} \frac{\Gamma(c) \Gamma(c-a-b)}{\Gamma(c-a) \Gamma(c-b)} \\
& +\widetilde{C}_{2}\left(\frac{1}{1-Q}\right)^{\gamma^{\prime}-\gamma}(1-Q-y)^{\gamma^{\prime}} \frac{\Gamma(c) \Gamma(a+b-c)}{\Gamma(a) \Gamma(b)} \\
& =\widetilde{C}_{1} e^{-\gamma \ln \left(\frac{1}{1-Q-y}\right)} \frac{\Gamma(c) \Gamma(c-a-b)}{\Gamma(c-a) \Gamma(c-b)}
\end{aligned}
$$

$$
\begin{aligned}
& +\widetilde{C}_{2}\left(\frac{1}{1-Q}\right)^{\gamma^{\prime}-\gamma} e^{-(1 / 2-\gamma) \ln \left(\frac{1}{1-Q-y}\right)} \\
& \times \frac{\Gamma(c) \Gamma(a+b-c)}{\Gamma(a) \Gamma(b)},
\end{aligned}
$$

with $\widetilde{C}_{1}=C_{1}(-1+Q)^{\alpha} / Q^{\alpha+\gamma}$ and $\widetilde{C}_{2}=C_{1}(-1+Q)^{\alpha} /$ $Q^{\alpha+\gamma^{\prime}}$. So, in order to have only outgoing waves at the cosmological horizon, we must impose $c-a=-n$ or $c-b=-n$, with $n=0,1,2 \ldots$. These conditions yield the following set of quasinormal frequencies:

$\omega= \pm \frac{\left(r_{-}-r_{+}\right) \sqrt{|A|} m}{2}-i \frac{\left(r_{-}-r_{+}\right)|A|}{4}(1+2 n)$.

Due to the imaginary part of the QNFs being negative the asymptotically $d S$ new type black holes are stable under fermionic perturbations, at least for the mode with the lowest angular momentum. Like in the previous case, the quasinormal frequencies associated to $\psi_{2}$ can be obtained; note that $\psi_{2}$ satisfies a similar equation to $\psi_{1}$ but making the changes $\kappa \rightarrow-\kappa$ and $\omega \rightarrow-\omega$.

3.2 Massless Dirac QNMs of asymptotically $d S$ and asymptotically locally flat new type black holes

In this section we compute the QNMs for asymptotically $d S$ $(A<0)$ and asymptotically locally flat $(A=0)$ new type black holes for massless fermionic perturbations $(m=0)$.

\subsubsection{Asymptotically dS new type black holes}

Performing the change of variables $y=1-\frac{r_{+}}{r}$, Eq. (16) reduces to

$$
\begin{aligned}
& y(y-1+Q) \psi_{1}^{\prime \prime}(y)+\frac{1}{2}(2 y-1+Q) \psi_{1}^{\prime}(y) \\
& +\left(-\frac{\kappa^{2} Q}{A r_{+}^{2}}+\frac{\omega^{2} Q^{2}}{A^{2} r_{+}^{2} y(y-1+Q)}\right. \\
& \left.-\frac{i \omega Q}{2 A r_{+}}\left(\frac{1}{y-1+Q}+\frac{1}{y}\right)\right) \times \psi_{1}=0,
\end{aligned}
$$

where we have defined $Q=r_{+} / r_{-}<1$. It is worth mentioning that in these coordinates the event horizon is located at $y=0$, and the cosmological horizon at $y=1-Q$. Now, making the change of variables

$\psi_{1}^{\prime \prime}(y)=y^{\alpha}(y-1+Q)^{\beta} F(y)$,

we arrive at the following equation:

$$
\begin{aligned}
& y(y-1+Q) F^{\prime \prime}(y)+\frac{1}{2}((Q-1)(1+4 \alpha) \\
& +2 y(1+2 \alpha+2 \beta)) F^{\prime}(y) \\
& +\left(-\frac{\kappa^{2} Q}{A r_{+}^{2}}+(\alpha+\beta)^{2}\right) F(y)=0
\end{aligned}
$$


where $\alpha$ and $\beta$ are given by

$\alpha_{+}=\frac{i Q \omega}{A r_{+}(1-Q)}, \quad \alpha_{-}=\frac{1}{2}-\frac{i Q \omega}{A r_{+}(1-Q)}$,

$\beta_{+}=-\frac{i Q \omega}{A r_{+}(1-Q)}, \quad \beta_{-}=\frac{1}{2}+\frac{i Q \omega}{A r_{+}(1-Q)}$.

In the following, we will use the values $\alpha=\frac{i Q \omega}{A r_{+}(1-Q)}$ and $\beta=-\frac{i Q \omega}{A r_{+}(1-Q)}$. Then, performing another change of variable, $(1-Q) z=y$, we obtain

$$
\begin{aligned}
& z(1-z) F^{\prime \prime}(z)+\left(\frac{1}{2}+2 \alpha-z(1+2 \alpha+2 \beta)\right) F^{\prime}(z) \\
& +\left(\frac{\kappa^{2} Q}{A r_{+}^{2}}-(\alpha+\beta)^{2}\right) F(z)=0
\end{aligned}
$$

and in these new coordinates the event horizon is located at $z=0$, and the cosmological horizon at $z=1$. We recognize Eq. (48) as the hypergeometric equation

$z(1-z) F^{\prime \prime}(z)+(c-(1+a+b) z) F^{\prime}(z)-a b F(z)=0$,

where

$$
\begin{aligned}
& a=\alpha+\beta \pm \frac{i \kappa \sqrt{Q}}{\sqrt{|A|} r_{+}}, \\
& b=\alpha+\beta \mp \frac{i \kappa \sqrt{Q}}{\sqrt{|A|} r_{+}}, \\
& c=\frac{1}{2}+2 \alpha .
\end{aligned}
$$

The general solution of the hypergeometric equation is

$$
\begin{aligned}
& F(z)=C_{12} F_{1}(a, b, c ; z) \\
& \quad+C_{2} z^{1-c}{ }_{2} F_{1}(a-c+1, b-c+1,2-c ; z),
\end{aligned}
$$

which has three regular singular points at $z=0, z=1$, and $z=\infty$. Here, ${ }_{2} F_{1}(a, b, c ; z)$ is a hypergeometric function and $C_{1}, C_{2}$ are constants. Then the solution for the radial function $\psi_{1}(z)$ is

$$
\begin{aligned}
& \psi_{1}(z)=C_{1} z^{\alpha}(1-z)_{2}^{\beta} F_{1}(a, b, c ; z) \\
& +C_{2} z^{1 / 2-\alpha}(1-z)_{2}^{\beta} F_{1}(a-c+1, b-c+1,2-c ; z) .
\end{aligned}
$$

So, in the vicinity of the event horizon $z=0$, and using the property ${ }_{2} F_{1}(a, b, c ; 0)=1$, the function $\psi_{1}(z)$ behaves as

$$
\psi_{1}(z)=C_{1} e^{\alpha \ln z}+C_{2} e^{(1 / 2-\alpha) \ln z} .
$$

Now, imposing the boundary conditions at the event horizon such that there are only ingoing modes implies that $C_{2}=0$. Thus, the solution can be written as

$$
\psi_{1}(z)=C_{1} z^{\alpha}(1-z)^{\beta}{ }_{2} F_{1}(a, b, c ; z) .
$$

On the other hand, using Kummer's formula for hypergeometric functions,

$$
\begin{aligned}
& { }_{2} F_{1}(a, b, c ; z)=\frac{\Gamma(c) \Gamma(c-a-b)}{\Gamma(c-a) \Gamma(c-b)} \\
& \quad \times{ }_{2} F_{1}(a, b, a+b-c ; 1-z) \\
& \quad+(1-z)^{c-a-b} \frac{\Gamma(c) \Gamma(a+b-c)}{\Gamma(a) \Gamma(b)} \\
& \quad \times{ }_{2} F_{1}(c-a, c-b, c-a-b+1 ; 1-z),
\end{aligned}
$$

the radial function $\psi_{1}(z)$ can be written as

$$
\begin{aligned}
\psi_{1}(z \rightarrow 1)= & C_{1}(1-z)^{\beta} \frac{\Gamma(c) \Gamma(c-a-b)}{\Gamma(c-a) \Gamma(c-b)} \\
& +C_{1}(1-z)^{1 / 2-\beta} \frac{\Gamma(c) \Gamma(a+b-c)}{\Gamma(a) \Gamma(b)} \\
= & C_{1} e^{-\beta \ln \left(\frac{1}{1-z}\right)} \frac{\Gamma(c) \Gamma(c-a-b)}{\Gamma(c-a) \Gamma(c-b)} \\
& +C_{1} e^{(\beta-1 / 2) \ln \left(\frac{1}{1-z}\right) \frac{\Gamma(c) \Gamma(a+b-c)}{\Gamma(a) \Gamma(b)}} .
\end{aligned}
$$

So, in order to have only outgoing waves at the cosmological horizon $z=1$, we must impose $c-a=-n$ or $c-b=-n$, with $n=0,1,2, \ldots$ These conditions yield the following set of quasinormal frequencies:

$\omega= \pm \frac{(1-Q) \sqrt{|A|} \kappa}{2 \sqrt{Q}}-i \frac{(1-Q)|A| r_{+}}{4 Q}(1+2 n)$.

In a similar way the quasinormal frequencies associated to $\psi_{2}$ can be obtained; note that $\psi_{2}$ satisfies a similar equation to $\psi_{1}$ but making the changes $\kappa \rightarrow-\kappa$ and $\omega \rightarrow-\omega$.

\subsubsection{Asymptotically locally flat new type black holes}

Under the change of variables $y=1-\frac{r_{+}}{r}$, Eq. (16) becomes

$$
\begin{aligned}
& y(y-1) \psi_{1}^{\prime \prime}(y)+\frac{1}{2}(2 y-1) \psi_{1}^{\prime}(y) \\
& +\left(\frac{\kappa^{2}}{B r_{+}}+\frac{\omega^{2}}{B^{2} y(y-1)}+\frac{i \omega}{2 B}\left(\frac{1}{y-1}+\frac{1}{y}\right)\right)=0 .
\end{aligned}
$$

Now, making the change of variables

$\psi_{1}^{\prime \prime}(y)=y^{\alpha}(y-1)^{\beta} F(y)$,

we arrive at the following equation:

$$
\begin{aligned}
& y(1-y) F^{\prime \prime}(y)+\frac{1}{2}(1+4 \alpha-2 y(1+2 \alpha+2 \beta)) F^{\prime}(y) \\
& +\left(-\frac{\kappa^{2}}{B r_{+}}-(\alpha+\beta)^{2}\right) F(y)=0
\end{aligned}
$$


where $\alpha$ and $\beta$ are given by

$\alpha_{+}=\frac{1}{2}+\frac{i \omega}{B}, \quad \alpha_{-}=-\frac{i \omega}{B}$,

$\beta_{+}=\frac{i \omega}{B}, \quad \beta_{-}=\frac{1}{2}-\frac{i \omega}{B}$.

In the following, we will use the values $\alpha=-\frac{i \omega}{B}$ and $\beta=\frac{i \omega}{B}$.

We recognize Eq. (60) as the hypergeometric equation

$y(1-y) F^{\prime \prime}(y)+(c-(1+a+b) y) F^{\prime}(y)-a b F(y)=0$,

where

$a=\alpha+\beta \pm \frac{i \kappa}{\sqrt{B r_{+}}}$,

$b=\alpha+\beta \mp \frac{i \kappa}{\sqrt{B r_{+}}}$,

$c=\frac{1}{2}+2 \alpha$.

As in the previous case, the general solution of the hypergeometric equation is

$$
\begin{aligned}
& F(y)=C_{12} F_{1}(a, b, c ; y) \\
& \quad+C_{2} y^{1-c}{ }_{2} F_{1}(a-c+1, b-c+1,2-c ; y),
\end{aligned}
$$

which has three regular singular points, at $y=0, y=1$, and $y=\infty$. Here, ${ }_{2} F_{1}(a, b, c ; y)$ is a hypergeometric function and $C_{1}, C_{2}$ are constants. Then the solution for the radial function $\psi_{1}(y)$ is

$$
\begin{aligned}
& \psi_{1}(y)=C_{1} y^{\alpha}(1-y)_{2}^{\beta} F_{1}(a, b, c ; y) \\
& \quad+C_{2} y^{1 / 2-\alpha}(1-y)^{\beta}{ }_{2} F_{1}(a-c+1, b-c+1,2-c ; y) .
\end{aligned}
$$

So, in the vicinity of the event horizon, $y=0$ and using the property ${ }_{2} F_{1}(a, b, c ; 0)=1$, the function $\psi_{1}(y)$ behaves as $\psi_{1}(z)=C_{1} e^{\alpha \ln y}+C_{2} e^{(1 / 2-\alpha) \ln y}$.

Now, imposing boundary conditions at the horizon such that there are only ingoing modes implies that $C_{2}=0$. Thus, the solution can be written as

$\psi_{1}(y)=C_{1} z^{\alpha}(1-y)^{\beta}{ }_{2} F_{1}(a, b, c ; y)$.

On the other hand, using Kummer's formula for hypergeometric functions (55), the radial function $\psi_{1}(y)$ can be written as

$$
\begin{aligned}
\psi_{1}(y \rightarrow 1)= & C_{1}(1-y)^{\beta} \frac{\Gamma(c) \Gamma(c-a-b)}{\Gamma(c-a) \Gamma(c-b)} \\
& +C_{1}(1-y)^{1 / 2-\beta} \frac{\Gamma(c) \Gamma(a+b-c)}{\Gamma(a) \Gamma(b)} \\
& =C_{1} e^{-\beta \ln \left(\frac{1}{1-y}\right)} \frac{\Gamma(c) \Gamma(c-a-b)}{\Gamma(c-a) \Gamma(c-b)} \\
& +C_{1} e^{(\beta-1 / 2) \ln \left(\frac{1}{1-y}\right) \frac{\Gamma(c) \Gamma(a+b-c)}{\Gamma(a) \Gamma(b)} .}
\end{aligned}
$$

So, in order to have only outgoing waves at the spatial infinity $y=1$, we must impose $c-a=-n$ or $c-b=-n$, with $n=0,1,2, \ldots$ These conditions yield the following set of quasinormal frequencies:

$\omega= \pm \frac{\kappa}{2} \sqrt{\frac{B}{r_{+}}}-i \frac{B}{4}(1+2 n)$.

\subsection{Massless Dirac QNMs of extremal new type black holes}

In this section we consider the extremal case $r_{+}=r_{-}(A>$ $0)$. First, we take $\kappa=0$. So, using the change of variable $y=1-\frac{r_{+}}{r}$ as in the previous sections, Eq. (16) reduces to

$$
\begin{aligned}
& y(1-y) \psi_{1}^{\prime \prime}(y)+(1-2 y) \psi_{1}^{\prime}(y) \\
& +\left(-\frac{m^{2}}{A y(1-y)}-\frac{i \omega}{A r_{+} y^{2}}+\frac{\omega^{2}(1-y)}{A^{2} r_{+}^{2} y^{3}}\right) \psi_{1}(y)=0 .
\end{aligned}
$$

The solution is given in terms of Whittaker functions,

$$
\begin{gathered}
\psi_{1}(y)=C_{1} \sqrt{\frac{y}{1-y}} \text { Whittaker } M\left(\frac{1}{2}, \frac{m}{\sqrt{A}},-\frac{2 i \omega(1-y)}{A r_{+} y}\right) \\
+C_{2} \sqrt{\frac{y}{1-y}} \text { Whittaker } W\left(\frac{1}{2}, \frac{m}{\sqrt{A}},-\frac{2 i \omega(1-y)}{A r_{+} y}\right) .
\end{gathered}
$$

In order to have a regular scalar field at spatial infinity $y \rightarrow 1$, we must set $C_{2}=0$. Therefore, the solution reduces to

$\psi_{1}(y)=C_{1} \sqrt{\frac{y}{1-y}}$ Whittaker $M\left(\frac{1}{2}, \frac{m}{\sqrt{A}},-\frac{2 i \omega(1-y)}{A r_{+} y}\right)$.

However, for $y \rightarrow 1$ this expression becomes null, which shows the absence of QNMs. Furthermore, for $m=0$ we obtain the equation

$$
\begin{aligned}
& y(1-y) \psi_{1}^{\prime \prime}(y)+(1-y) \psi_{1}^{\prime}(y) \\
& +\left(-\frac{\kappa^{2}(1-y)}{A r_{+}^{2} y}+\frac{i \omega}{A r_{+} y}-\frac{i \omega}{A r_{+} y^{2}}+\frac{\omega^{2}(1-y)}{A^{2} r_{+}^{2} y^{3}}\right) \\
& \quad \times \psi_{1}(y)=0
\end{aligned}
$$

whose solution is given in terms of confluent Heun functions

$$
\begin{aligned}
& \psi_{1}(y)=C_{1} e^{i \omega /\left(A r_{+} y\right)} y^{\kappa / \sqrt{A} r_{+} \text {Heun }_{C}} \\
& \times\left(\frac{2 i \omega}{A r_{+}},-\frac{2 \kappa}{\sqrt{A} r_{+}},-1,-\frac{i \omega}{A r_{+}}, \frac{i \omega}{A r_{+}}+\frac{1}{2}, \frac{1}{y}\right) \\
& +C_{2} e^{i \omega /\left(A r_{+} y\right)} y^{-\kappa / \sqrt{A} r_{+}} \text {Heun }_{C} \\
& \times\left(\frac{2 i \omega}{A r_{+}}, \frac{2 \kappa}{\sqrt{A} r_{+}},-1,-\frac{i \omega}{A r_{+}}, \frac{i \omega}{A r_{+}}+\frac{1}{2}, \frac{1}{y}\right) .
\end{aligned}
$$


Then, at infinity $y \rightarrow 1$, the radial function vanishes. Therefore, there are not quasinormal modes for extremal new type black holes.

\section{Conclusions}

In this work we have calculated analytically the QNMs of fermionic perturbations for some special cases for a new type of black holes, which are solutions of three-dimensional NMG and also are solutions of conformal gravity in three dimensions. The first case that we have analyzed concerns massive fermionic fields perturbations without angular momentum $(\kappa=0)$ in the black hole background, and we have found the QNFs for an asymptotically $A d S(d S)$ new type of black holes. For asymptotically $A d S$ new type black holes the QNFs are purely imaginary and negative, which ensures the stability of the black hole under fermionic perturbations. For an asymptotically $d S$ new type black holes the QNFs have a real and imaginary part, and the imaginary part is negative, which ensures the stability of the black hole under fermionic perturbations. It is worth mentioning that in these cases the Dirac equation can be written as a Riemann differential equation, as in [46]. Other cases, where is possible to find the QNFs analytically, are for asymptotically $d S$ and asymptotically locally flat new types of black holes for massless fermionic field perturbations; in these cases we have found that the QNFs have a real and imaginary part, and the imaginary part is negative, which ensures the stability of the black hole under fermionic perturbations.

Finally, we have analyzed fermionic field perturbations in the extremal new type black holes for some special cases, and we found that there are not quasinormal modes as occurs, for instance, in [46,47], where the authors have showed the absence of QNMs in the extremal BTZ black hole and the extremal four-dimensional Lifshitz Black Hole in Conformal Gravity. However, it was shown that it is possible to construct the QNMs of three-dimensional extremal black holes in an algebraic way as the descendants of the highest weight modes [48], with hidden conformal symmetry being an intrinsic property of the extremal black hole. Also, it is worth to mention that the absence of QNMs for extremal black holes does not always occur, for instance see [49], where the authors have showed the presence of QNMs for the extremal BTZ black holes in TMG.

\begin{abstract}
Acknowledgments We thank to Julio Oliva for useful comments. This work was funded by the Comisión Nacional de Investigación Científica y Tecnológica through FONDECYT Grant 11121148 (Y.V.). P. A. G. acknowledges the hospitality of the Universidad de La Serena where part of this work was undertaken.
\end{abstract}

Open Access This article is distributed under the terms of the Creative Commons Attribution License which permits any use, distribution, and reproduction in any medium, provided the original author(s) and the source are credited.

Funded by $\mathrm{SCOAP}^{3}$ / License Version CC BY 4.0.

\section{References}

1. M. Banados, C. Teitelboim, J. Zanelli, Phys. Rev. Lett. 69, 1849 (1992). hep-th/9204099

2. S. Deser, R. Jackiw, S. Templeton, Ann. Phys. 140, 372 (1982) [Erratum-ibid. 185, 406 (1988)]

3. S. Deser, R. Jackiw, S. Templeton, Ann. Phys. 185, 406 (1988)

4. S. Deser, R. Jackiw, S. Templeton, Ann. Phys. 281, 409 (2000)

5. S. Deser, R. Jackiw, S. Templeton, Phys. Rev. Lett. 48, 975 (1982)

6. E.A. Bergshoeff, O. Hohm, P.K. Townsend, Phys. Rev. Lett. 102, 201301 (2009). arXiv:0901.1766 [hep-th]

7. E.A. Bergshoeff, O. Hohm, P.K. Townsend, Ann. Phys. 325, 1118 (2010). arXiv:0911.3061 [hep-th]

8. E. Bergshoeff, O. Hohm, P. Townsend, J. Phys. Conf. Ser. 229, 012005 (2010). arXiv:0912.2944 [hep-th]

9. R. Andringa, E.A. Bergshoeff, M. de Roo, O. Hohm, E. Sezgin, P.K. Townsend, Class. Quant. Grav. 27, 025010 (2010). arXiv:0907.4658 [hep-th]

10. E.A. Bergshoeff, O. Hohm, J. Rosseel, E. Sezgin, P.K. Townsend, Class. Quant. Grav. 28, 015002 (2011). arXiv:1005.3952 [hep-th]

11. M. Nakasone, I. Oda, Prog. Theor. Phys. 121, 1389 (2009). arXiv:0902.3531 [hep-th]

12. G. Clement, Class. Quant. Grav. 26, 105015 (2009). arXiv:0902.4634 [hep-th]

13. E. Ayon-Beato, G. Giribet, M. Hassaine, JHEP 0905, 029 (2009). arXiv:0904.0668 [hep-th]

14. G. Clement, Class. Quant. Grav. 26, 165002 (2009). arXiv:0905.0553 [hep-th]

15. E. Ayon-Beato, A. Garbarz, G. Giribet, M. Hassaine, Phys. Rev. D 80, 104029 (2009). arXiv:0909.1347 [hep-th]

16. J. Oliva, D. Tempo, R. Troncoso, JHEP 0907, 011 (2009). arXiv:0905.1545 [hep-th]

17. E.A. Bergshoeff, O. Hohm, P.K. Townsend, Phys. Rev. D 79, 124042 (2009). arXiv:0905.1259 [hep-th]

18. W. Kim, E.J. Son, Phys. Lett. B 678, 107 (2009). arXiv:0904.4538 [hep-th]

19. I. Oda, JHEP 0905, 064 (2009). arXiv:0904.2833 [hep-th]

20. Y. Liu, Y.-W. Sun, Phys. Rev. D 79, 126001 (2009). arXiv:0904.0403 [hep-th]

21. M. Nakasone, I. Oda, Phys. Rev. D 79, 104012 (2009). arXiv:0903.1459 [hep-th]

22. S. Deser, Phys. Rev. Lett. 103, 101302 (2009). arXiv:0904.4473 [hep-th]

23. F. Correa, M. Hassaine, J. Oliva, Phys. Rev. D 89, 124005 (2014). arXiv:1403.6479 [hep-th]

24. T. Regge, J.A. Wheeler, Phys. Rev. 108, 1063 (1957)

25. F.J. Zerilli, Phys. Rev. D 2, 2141 (1970)

26. F.J. Zerilli, Phys. Rev. Lett. 24, 737 (1970)

27. K. D. Kokkotas, B.G. Schmidt, Living Rev. Rel. 2, 2 (1999). gr-qc/9909058

28. H.-P. Nollert, Class. Quant. Grav. 16, R159 (1999)

29. R.A. Konoplya, A. Zhidenko, Rev. Mod. Phys. 83, 793 (2011). arXiv: 1102.4014 [gr-qc]

30. J.S.F. Chan, R.B. Mann, Phys. Rev. D 55, 7546 (1997). gr-qc/9612026

31. V. Cardoso, J.P.S. Lemos, Phys. Rev. D 63, 124015 (2001). gr-qc/0101052

32. D. Birmingham, I. Sachs, S.N. Solodukhin, Phys. Rev. Lett. 88, 151301 (2002). hep-th/0112055 
33. Y. Kwon, S. Nam, J.-D. Park, S.-H. Yi, Class. Quant. Grav. 28, 145006 (2011). arXiv:1102.0138 [hep-th]

34. J.M. Maldacena, Adv. Theor. Math. Phys. 2, 231 (1998). hep-th/9711200

35. G.T. Horowitz, V.E. Hubeny, Phys. Rev. D 62, 024027 (2000). hep-th/9909056

36. J.D. Bekenstein, Lett. Nuovo Cim. 11, 467 (1974)

37. S. Hod, Phys. Rev. Lett. 81, 4293 (1998). gr-qc/9812002

38. G. Kunstatter, Phys. Rev. Lett. 90, 161301 (2003). gr-qc/0212014

39. M. Maggiore, Phys. Rev. Lett. 100, 141301 (2008). arXiv:0711.3145 [gr-qc]

40. C. Corda, Int. J. Mod. Phys. D 21, 1242023 (2012). arXiv:1205.5251 [gr-qc]

41. C. Corda, Eur. Phys. J. C 73, 2665 (2013). arXiv:1210.7747 [gr-qc]

42. C. Corda, S.H. Hendi, R. Katebi, N.O. Schmidt, JHEP 1306, 008 (2013). arXiv:1305.3710 [gr-qc]
43. C. Corda, S.H. Hendi, R. Katebi, N.O. Schmidt, Adv. High Energy Phys. 2014, 527874 (2014). arXiv:1401.2872 [physics.gen-ph]

44. J. Oliva, D. Tempo, R. Troncoso, Int. J. Mod. Phys. A 24, 1588 (2009). arXiv:0905.1510 [hep-th]

45. M. Abramowitz, A. Stegun, Handbook of Mathematical Functions (Dover Publications, New York, 1970)

46. M. Catalan, E. Cisternas, P.A. Gonzalez, Y. Vasquez, arXiv:1404.3172 [gr-qc]

47. J. Crisostomo, S. Lepe, J. Saavedra, Class. Quant. Grav. 21, 2801 (2004). hep-th/0402048

48. B. Chen, J.-J. Zhang, Phys. Lett. B 699, 204 (2011). arXiv:1012.2219 [hep-th]

49. H.R. Afshar, M. Alishahiha, A.E. Mosaffa, JHEP 1008, 081 (2010). arXiv:1006.4468 [hep-th] 